\title{
Existence results for an impulsive neutral integro-differential equations in Banach spaces
}

\author{
Venkatesh Usha, Dumitru Baleanu and Mani Mallika Arjunan
}

\begin{abstract}
In this manuscript we investigate the existence of mild solution for a abstract impulsive neutral integro-differential equation by using semigroup theory and Krasnoselskii-Schaefer fixed point theorem in different approach. At last, an example is also provided to illustrate the obtained results.
\end{abstract}

\section{Introduction}

The systems of differential equations with impulses can be successfully applied to model the shutter dynamics of a blow back valve. This is the common example for mathematical model in impulsive differential equation. An important specific feature that differentiate the differential equation without impulses and impulsive differential equations is the fact that their solutions are subjected to the multiple external influences, which are discrete in time and their solutions are discontinuous functions. This type of equations are well studied due to their wide application in practice. Nowadays the theory of impulsive differential and partial differential equations have become an important area of investigation because of its applicability is wide in biological systems, blood flows, population dynamics, control, mechanics, electrical engineering fields, mechanical systems with impact, theoretical physics, radio

Key Words: Fixed point theorem, Differential equations with Impulses, Neutral equations, Semigroups of linear operators

2010 Mathematics Subject Classification: Primary 47H10, 34A37; Secondary 34K40, 47D03.

Received: 18.01.2019

Accepted: 15.03.2019 
EXISTENCE RESULTS FOR AN IMPULSIVE NEUTRAL

physics, pharmacokinetics, mathematical economy, chemical technology, electric technology, industrial robotics, metallurgy, ecology, medicine and so on. We refer the monographs of Benchora et al. [4], Lakshmikantham et al. [19] and the papers of $[5,7,8,22,24,23,11]$ and the references cited therein for more details on this theory and on its applications.

Recently, the theory of impulsive differential equations as much as neutral differential equations represents an important area of investigation having various applications to problems arising in mechanics, electrical engineering, biology, ecology and in many areas of applied mathematics. The system of rigid heat conduction with finite wave spaces were described in the form of the integro-differential equation of neutral type with delay. Recently neutral impulsive differential and integro-differential equations have received substantial interest from researchers $[7,17,9,10,1,2,20]$. Since the literature regarding to ordinary neutral differential equations is quite extensive, we suggest the reader to refer Hale [12], which has a comprehensive presentation on these equations. For further applications of partial functional differential equations of neutral type, we refer to Hale [13] and $\mathrm{Wu}[25,26,27]$.

In recent years the theory of semigroups of bounded linear operator together with differential and integro-differential equations has been extended to a large and interesting theory of semigroups of nonlinear operators in Hilbert and Banach Spaces. Some more recent results we applied on semigroups can be found in Pazy [21]. The fractional powers of operators and their properties were investigated by Pazy [21]. Alka Chadha et al. [6] established the mild solutions for an impulsive neutral integro-differential equation with infinite delay via fractional operators.

As the generalization of abstract impulsive neutral integro differential equations have attracted the researchers in a great manner. However Langing $\mathrm{Hu}$ et al. [18] discussed about the solutions for a class of impulsive neutral stochastic functional integro-differential equations with infinite delay in an abstract space by means of the Krasnoselskii-Schaefer type fixed point theorem. In [7], the existence of mild solutions for impulsive neutral functional differential equations with infinite delay was examined in Banach spaces and Balachandran et al. [3] discussed the existence of mild solutions for impulsive neutral evolution integro-differential equations with infinite delay in same space by using analytic semigroup theory and the Krasnoselskii-Schaefer type fixed point theorem with examples.

Concerning general motivations, relevant developments and the current status of the theory of abstract impulsive differential equations, we suggest $[14,15,16,17]$. Therefore, the underlying commitment for the advancement of theory on the existence and qualitative properties of solutions for abstract neutral differential system described in the abstract form and their specialized 
EXISTENCE RESULTS FOR AN IMPULSIVE NEUTRAL

approach permits some notable simplifications in the study of other classes of abstract neutral differential equations.

By the above mentioned motivations, we analyzed the existence of mild solutions for an abstract impulsive neutral integro differential equations by using fixed point technique. This paper has four sections. In Section 2, we recall the notations, definitions and lemmas which are used throughout this paper. In Section 3, we study the existence of mild solutions of the problem (2.1) - (2.3) by using Banach contraction principle and Krasnoselskii-Schaefer type fixed point theorem. In section 4 , we describe an application to impulsive partial differential equations.

\section{Problem formation and preliminaries}

Below, we present some preliminary results and definitions to prove the main results. First we consider the abstract impulsive neutral integro-differential equation of the model

$$
\begin{aligned}
\frac{d}{d t}[u(t)+ & \left.g\left(t, u(t), \int_{0}^{t} e_{1}(t, s, u(s)) d s\right)\right] \\
& =A\left[u(t)+g\left(t, u(t), \int_{0}^{t} e_{1}(t, s, u(s)) d s\right)\right] \\
+ & f\left(t, u(t), \int_{0}^{t} e_{2}(t, s, u(s)) d s\right), t \in(0, a], t \neq t_{i}, i=1, \ldots N \\
u(0) & =u_{0} \in X \\
\Delta u\left(t_{i}\right) & =I_{i}\left(u\left(t_{i}\right)\right), i=1,2, \ldots N
\end{aligned}
$$

where $A$ is the infinitesimal generator of an analytic semigroup $\{T(t)\}_{t \geq 0}$ in a Banach space $X$ having norm $\|\cdot\|$ and $M_{1}$ is a positive constant to ensure that $\|T(t)\| \leq M_{1}$, the domain of the $\alpha$ - fractional power of $-A$ endowed with the graph norm $\left\|(-A)^{\alpha} x\right\|$ is denoted as $X_{\alpha}$ and $f, g:[0, a] \times X_{\alpha} \times X_{\alpha} \rightarrow$ $X, I_{i} \in C\left(X_{\alpha}, X\right)$ for all $i=1, \ldots, N$, are given appropriate functions and $e_{k}:[0, a] \times X_{\alpha} \rightarrow X, k=1,2$ are suitable functions. Due to the previously mentioned advancements, in our paper we present our outcomes of existence of mild solutions of an abstract impulsive differential equations. The type of function $I_{i}$ we considered in the problem (2.3) are mapped from $X_{\alpha}$ into $X$. And we follow $P C_{\alpha}\left(X_{\alpha}\right)$, the class of Banach spaces with weight formed by piecewise continuous functions. Here $J=\left[0=t_{0}<t_{1}<t_{2}<\cdots<t_{N}<a\right]$ are fixed numbers. $\Delta u\left(t_{i}\right)$ represents the jump of $u(\cdot)$ at $t_{i}$ which is denoted by $\Delta u\left(t_{i}\right)=u\left(t_{i}^{+}\right)+u\left(t_{i}^{-}\right)$, where $u\left(t_{i}^{+}\right)$and $u\left(t_{i}^{-}\right)$denote the right and left limit of $u(\cdot)$ at $t_{i}$ respectively. 
EXISTENCE RESULTS FOR AN IMPULSIVE NEUTRAL

Now we present some notations and techniques needed in the manuscript. Let $\left(\mathscr{Z},\|\cdot\|_{\mathscr{Z}}\right)$ and $\left(\mathscr{W},\|\cdot\|_{\mathscr{W}}\right)$ be Banach spaces. Below $\mathscr{L}(\mathscr{Z}, \mathscr{W})$ represents the space of bounded linear operators from $\mathscr{Z}$ into $\mathscr{W}$ with the norm of operators denoted by $\|\cdot\|_{\mathscr{L}(\mathscr{Z}, \mathscr{W})}$ and we depict $\mathscr{L}(\mathscr{Z})$ and $\|\cdot\|_{\mathscr{L}(\mathscr{Z})}$ such that $\mathscr{Z}=\mathscr{W}$. And also, the closed ball $B_{l}(z, \mathscr{Z})$ with center at $z$ and radius $l$ in $\mathscr{Z}$ and for an interval $J \subset \mathbb{R}$. For the space formed by all bounded continuous functions from $J$ into $\mathscr{Z}$, we consider the notation $C(J ; \mathscr{Z})$ equipped with the uniform norm depicted by $\|\cdot\|_{C(J ; X)}$.

Let $0 \in \varrho(A)$, then we can define the fractional power $(-A)^{\alpha}$ for $0<\alpha \leq 1$, as a closed linear operator on its domain $D\left((-A)^{\alpha}\right)$ and $\left\|(-A)^{\alpha}\right\| \leq M_{0}$. Then, the subspace $D\left((-A)^{\alpha}\right)$ is dense in $X$ and the norm defined in it is

$$
\|x\|_{\alpha}=\left\|(-A)^{\alpha} x\right\|, x \in D\left((-A)^{\alpha}\right) .
$$

Therefore from now we denote $X_{\alpha}$ be the Banach space $D\left((-A)^{\alpha}\right)$ normed with $\|X\|_{\alpha}$.

Proposition 2.1. [21] Let $\{T(t)\}_{t \leq 0}$ be an analytic semigroup with infinitesimal generator $A$, the following properties will be used:

(i) for $0<\alpha \leq 1, X_{\alpha}$ is a Banach space.

(ii) If $0<\beta<\alpha \leq 1$, then $X_{\alpha} \subset X_{\beta}$ and the imbedding is compact whenever the resolvent operator of $A$ is compact.

(iii) for any $0 \leq \alpha \leq 1$, there exists a positive constant $C_{\alpha}$ fulfilling

$$
\left\|(-A)^{\alpha} T(t)\right\| \leq \frac{C_{\alpha}}{t^{\alpha}}, 0<t \leq a .
$$

In this paper, $\alpha \in(0,1), t_{0}=0, t_{N+1}=a$ and $\delta_{i}=\left(t_{i+1}-t_{i}\right)$ where $i=0,1, \ldots, N$. We consider the space $P C(X)$ formed by all the functions $u:[0, a] \rightarrow X$ for impulsive conditions such that $u(\cdot)$ is continuous at $t \neq$ $t_{i}, u\left(t_{i}^{-}\right)=u\left(t_{i}\right)$ and $u\left(t_{i}^{+}\right)$exists for all $i=0,1, \ldots, N$, with the uniform norm defined in it. We consider the space $P C_{\alpha}\left(X_{\alpha}\right)$ formed by all the functions $u:(0, a] \rightarrow X_{\alpha}$ such that $u_{\mid\left(t_{i}, t_{i+1}\right]} \in C\left(\left(t_{i}, t_{i+1}\right] ; X_{\alpha}\right)$ and for all values of $i=0,1, \ldots, N$,

$$
\|u\|_{\alpha, i}=\sup _{t \in\left(t_{i}, t_{i+1}\right]}\left(t-t_{i}\right)^{\alpha}\left\|(-A)^{\alpha} u(t)\right\|<\infty
$$

with the norm

$$
\|u\|_{\alpha}=\max _{i=0,1, \ldots, N}\|u\|_{\alpha, i} .
$$


Then, $P C_{\alpha}\left(X_{\alpha}\right)$ is a Banach space. Again we consider the function $\widetilde{u_{i}} \in$ $C\left(\left[t_{i}, t_{i+1} ; X\right]\right)$ is given by

$$
\tilde{u}_{i}(t)=\left\{\begin{array}{l}
u(t) \text { for } t \in\left(t_{i}, t_{i+1}\right], \\
u\left(t_{i}^{+}\right) \text {for } t=t_{i} .
\end{array}\right.
$$

If $\mathscr{B} \subseteq P C(X)$, we use the set $\widetilde{\mathscr{B}}_{i}=\left\{\widetilde{u}_{i}: u \in \mathscr{B}\right\}$. We show the following lemma.

Lemma 2.1. A set $\mathscr{B} \subseteq P C(X)$ is relatively compact in $P C(X)$ if and only if $\widetilde{\mathscr{B}}_{i}$ is relatively compact in $C\left(\left[t_{i}, t_{i+1}\right], X\right)$ for all $i \in\{1, \ldots, N\}$.

In consistence with the prior specified discussion, we specify the mild solution of the model $(2.1)-(2.3)$.

Definition 2.1. A function $u \in P C(X)$ is called a mild solution of the model $(2.1)-(2.3)$ if

$$
u(t)=\left\{\begin{array}{c}
T(t)\left(u_{0}+g\left(0, u_{0}, 0\right)\right)-g\left(t, u(t), \int_{0}^{t} e_{1}(t, s, u(s)) d s\right) \\
+\int_{0}^{t} T(t-s) f\left(s, u(s), \int_{0}^{s} e_{2}(s, \tau, u(\tau)) d \tau\right) d s \\
+\sum_{t_{i}<t} T\left(t-t_{i}\right) I_{i}\left(u\left(t_{i}\right)\right), \quad \forall t \in J
\end{array}\right.
$$

For a number $q>1$, let $q^{\prime}$ be the conjugate of $q$, then $\frac{1}{q}+\frac{1}{q^{\prime}}=1$ and take $q^{\prime}=\infty$ for $q=1$.

We list the following hypotheses in order to investigate the existence of $(2.1)-(2.3)$

(H1) The function $g$ belongs to $C\left(J \times X_{\alpha} \times X_{\alpha} ; X\right), q \in[1,1 / \alpha)$ for $\alpha \in(0,1)$ and the functions $K_{g}$ and $\widetilde{K}_{g} \in L^{q^{\prime}}\left(J ; \mathbb{R}^{+}\right)$and $K_{g}^{*}>0$; fulfills the subsequent assumptions for all $t \in J$,

(i) $\left\|g\left(t, \vartheta_{1}, \sigma_{1}\right)-g\left(t, \vartheta_{2}, \sigma_{2}\right)\right\| \leq K_{g}(t)\left\|(-A)^{\alpha} \vartheta_{1}-(-A)^{\alpha} \vartheta_{2}\right\|$ $+\widetilde{K}_{g}(t)\left\|(-A)^{\alpha} \sigma_{1}-(-A)^{\alpha} \sigma_{2}\right\|, \vartheta_{k}, \sigma_{k} \in X_{\alpha} ;$ where $k=1,2$,

(ii) $\|g(t, \vartheta, 0)\| \leq K_{g}(t)\left\|(-A)^{\alpha} \vartheta\right\|+K_{g}^{*}$ and $K_{g}^{*}=\max _{t \in J}\|g(t, 0,0)\|$.

(H2) For $f \in C\left(J \times X_{\alpha} \times X_{\alpha} ; X\right)$ and $q \in[1,1 / \alpha), \alpha \in(0,1)$ and the functions $K_{f}$ and $\widetilde{K}_{f} \in L^{q^{\prime}}\left(J ; \mathbb{R}^{+}\right)$and $K_{f}^{*}>0$, for all $t \in J$ such that

$$
\begin{aligned}
& \left\|f\left(t, \varphi_{1}, \mu_{1}\right)-f\left(t, \varphi_{2}, \mu_{2}\right)\right\| \leq K_{f}(t)\left\|(-A)^{\alpha} \varphi_{1}-(-A)^{\alpha} \varphi_{2}\right\| \\
& +\widetilde{K}_{f}(t)\left\|(-A)^{\alpha} \mu_{1}-(-A)^{\alpha} \mu_{2}\right\|, \varphi_{k}, \mu_{k} \in X_{\alpha} ; k=1,2
\end{aligned}
$$

and $K_{f}^{*}=\max _{t \in J}\|f(t, 0,0)\|$. 
(H3) The functions $e_{k}: J \times X_{\alpha}$ are continuous and there exist constants $K_{e_{k}}>0, K_{e_{k}}^{*}>0$, such that

$$
\begin{aligned}
& \left\|e_{k}(t, s, \omega)-e_{k}(t, s, \psi)\right\| \leq K_{e_{k}}\|\omega-\psi\|, \omega, \psi \in X_{\alpha},(t, s) \in J, k=1,2 ; \\
& \qquad\left\|e_{k}(t, s, \omega)\right\| \leq K_{e_{k}}(t, s)\|\omega\|_{X_{\alpha}}, \\
& \text { and } K_{e_{k}}^{*}=\max _{t \in J} \int_{0}^{t} K_{e_{k}}(t, s) d s, k=1,2 .
\end{aligned}
$$

(H4) The function $I_{i} \in C\left(X_{\alpha} ; X\right)$ and the functions $K_{I_{i}}:[0, \infty) \rightarrow \mathbb{R}^{+}$, which are non-decreasing for all $\phi_{1}, \phi_{2}$ in $B_{l}\left(0, X_{\alpha}\right)$, for all $i=1,2, \ldots, N$ and each $l>0$, such that

$$
\left\|I_{i}\left(\phi_{1}\right)-I_{i}\left(\phi_{2}\right)\right\| \leq K_{I_{i}}(l)\left\|(-A)^{\alpha}\left(\phi_{1}-\phi_{2}\right)\right\| .
$$

\section{$3 \quad$ Existence of Mild Solutions}

In this area we show and illustrate the existence results for the model (2.1)(2.3) under Banach contraction principle and Krasnoselskii-Schaefer type fixed point theorem.

Theorem 3.1. Assume that the hypotheses $(H 1)-(H 4)$ hold and for $r>0$, $\Theta \in(0,1)$ such that

$$
\begin{gathered}
C_{\alpha}\left\{p_{1}+\Upsilon+\sum_{i=1}^{j} \frac{K_{I_{i}}\left(\frac{r}{\delta_{i-1}^{\alpha}}\right)}{\delta_{i-1}^{\alpha}}\right\} \leq \Theta r \\
\Omega=C_{\alpha}\left[\left\|g\left(0, u_{0}, 0\right)\right\|+p_{2}+p_{3} K_{f}^{*}+\sum_{i=1}^{j}\left\|I_{i}(0)\right\|\right]<(1-\Theta) r .
\end{gathered}
$$

If $\left\|u_{0}\right\| \leq \frac{((1-\Theta) r-\Omega)}{C_{\alpha}}$, then there exists a unique mild solution $u \in P C_{\alpha}\left(X_{\alpha}\right)$ of $(2.1)-(2.3)$.

Proof. Let the map $\Gamma: P C_{\alpha}\left(X_{\alpha}\right) \rightarrow P C_{\alpha}\left(X_{\alpha}\right)$ characterized by

$$
\begin{aligned}
\Gamma u(t)= & T(t)\left[u_{0}+g\left(0, u_{0}, 0\right)\right]-g\left(t, u(t), \int_{0}^{t} e_{1}(t, s, u(s)) d s\right) \\
& +\int_{0}^{t} T(t-s) f\left(s, u(s), \int_{0}^{s} e_{2}(s, \tau, u(\tau)) d \tau\right) d s \\
& +\sum_{t_{i}<t} T\left(t-t_{i}\right) I_{i}\left(u\left(t_{i}\right)\right), \quad \text { for all } t \in(0, a] .
\end{aligned}
$$

To show the map is a contraction on $B_{r}\left(0, P C_{\alpha}\left(X_{\alpha}\right)\right)$, initially we prove that $\Gamma$ has values in $B_{r}\left(0, P C_{\alpha}\left(X_{\alpha}\right)\right)$. 


$$
\begin{aligned}
& \left\|(-A)^{\alpha} \Gamma u(t)\right\|=\|(-A)^{\alpha} T(t)\left[u_{0}+g\left(0, u_{0}, 0\right)\right] \\
& -(-A)^{\alpha} g\left(t, u(t), \int_{0}^{t} e_{1}(t, s, u(s)) d s\right) \\
& +(-A)^{\alpha} \int_{0}^{t} T(t-s) f\left(s, u(s), \int_{0}^{s} e_{2}(s, \tau, u(\tau)) d \tau\right) d s \\
& +(-A)^{\alpha} \sum_{t_{i}<t} T\left(t-t_{i}\right) I_{i}\left(u\left(t_{i}\right)\right) \|, \quad \text { for all } t \in(0, a] . \\
& \leq\left\|(-A)^{\alpha} T(t)\left[u_{0}+g\left(0, u_{0}, 0\right)\right]\right\| \\
& +\left\|(-A)^{\alpha} g\left(t, u(t), \int_{0}^{t} e_{1}(t, s, u(s)) d s\right)\right\| \\
& +\left\|\int_{0}^{t}(-A)^{\alpha} T(t-s) f\left(s, u(s), \int_{0}^{s} e_{2}(s, \tau, u(\tau)) d \tau\right) d s\right\| \\
& +\left\|(-A)^{\alpha} \sum_{t_{i}<t}^{1} T\left(t-t_{i}\right) I_{i}\left(u\left(t_{i}\right)\right)\right\| \\
& =\sum_{i=1}^{4} J_{i} \\
& J_{1}=\left\|(-A)^{\alpha} T(t)\left(u_{0}+g\left(0, u_{0}, 0\right)\right)\right\| \\
& \leq\left\|(-A)^{\alpha} T(t)\left(u_{0}\right)\right\|+\left\|(-A)^{\alpha} T(t) g\left(0, u_{0}, 0\right)\right\| \\
& \leq \frac{C_{\alpha}}{t^{\alpha}}\left[\left\|u_{0}\right\|+\left\|g\left(0, u_{0}, 0\right)\right\|\right] \\
& \leq \frac{C_{\alpha}}{\left(t-t_{j}\right)^{\alpha}}\left[\left\|u_{0}\right\|+\left\|g\left(0, u_{0}, 0\right)\right\|\right] . \\
& J_{2}=\left\|(-A)^{\alpha} g\left(t, u(t), \int_{0}^{t} e_{1}(t, s, u(s)) d s\right)\right\| \\
& \leq\left\|(-A)^{\alpha}\right\|\left\|g\left(t, u(t), \int_{0}^{t} e_{1}(t, s, u(s)) d s\right)\right\| \\
& \leq \frac{C_{\alpha}\|u\|_{\alpha}}{\left(t-t_{j}\right)^{\alpha}}\left[M_{0} K_{g}(t) \frac{\left(t-t_{j}\right)^{\alpha}}{C_{\alpha}\left(t-t_{i}\right)^{\alpha}}+M_{0} \widetilde{K}_{g}(t) K_{e_{1}}^{*} \frac{\left(t-t_{j}\right)^{\alpha}}{C_{\alpha}\left(t-t_{i}\right)^{\alpha}}\right] \\
& \leq \frac{C_{\alpha}\|u\|_{\alpha} p_{1}}{\left(t-t_{j}\right)^{\alpha}}+\frac{C_{\alpha} p_{2}}{\left(t-t_{j}\right)^{\alpha}} \\
& \text { where } p_{1}=\left[M_{0} K_{g}(t) \frac{\left(t-t_{j}\right)^{\alpha}}{C_{\alpha}\left(t-t_{i}\right)^{\alpha}}+M_{0} \widetilde{K}_{g}(t) K_{e_{1}}^{*} \frac{\left(t-t_{j}\right)^{\alpha}}{C_{\alpha}\left(t-t_{i}\right)^{\alpha}}\right]
\end{aligned}
$$


EXISTENCE RESULTS FOR AN IMPULSIVE NEUTRAL

$$
\text { and } p_{2}=M_{0} \frac{\left(t-t_{j}\right)^{\alpha}}{C_{\alpha}} K_{g}^{*} \text {. }
$$

On the other hand, from the Hölder's inequality, Lemmas (2.1), (2.2) of [14] and by the proposition (2.1), we have

$$
\begin{aligned}
& J_{3}=\left\|\int_{0}^{t}(-A)^{\alpha} T(t-s) f\left(s, u(s), \int_{0}^{s} e_{2}(s, \tau, u(\tau)) d \tau\right) d s\right\| \\
& \leq \int_{0}^{t}\left\|(-A)^{\alpha} T(t-s)\right\|\left\|f\left(s, u(s), \int_{0}^{s} e_{2}(s, \tau, u(\tau)) d \tau\right)-f(s, 0,0)\right\| \\
& \quad+\|f(s, 0,0)\| d s \\
& \leq \int_{0}^{t} \frac{C_{\alpha}}{(t-s)^{\alpha}}\left[K_{f}(s)\left\|(-A)^{\alpha} u(s)\right\|+\widetilde{K}_{f}(s)\left\|(-A)^{\alpha} \int_{0}^{s} e_{2}(s, \tau, u(\tau)) d \tau\right\|\right. \\
&\quad+\|f(s, 0,0)\|] d s \\
& \leq \int_{0}^{t} \frac{C_{\alpha}}{(t-s)^{\alpha}} K_{f}(s)\left\|(-A)^{\alpha} u(s)\right\| d s+\int_{0}^{t} \frac{C_{\alpha}}{(t-s)^{\alpha}} \widetilde{K}_{f}(s) \\
& \quad \times\left\|(-A)^{\alpha} \int_{0}^{s} e_{2}(s, \tau, u(\tau)) d \tau\right\| d s+\int_{0}^{t} \frac{C_{\alpha}}{(t-s)^{\alpha}}\|f(s, 0,0)\| d s \\
& \quad J_{3}=Q_{1}+Q_{2}+Q_{3},
\end{aligned}
$$

where

$$
\begin{aligned}
Q_{1} & =C_{\alpha} \int_{0}^{t} \frac{K_{f}(s)}{(t-s)^{\alpha}}\left\|(-A)^{\alpha} u(s)\right\| d s, \\
Q_{2} & =C_{\alpha} \int_{0}^{t} \frac{\widetilde{K}_{f}(s)}{(t-s)^{\alpha}}\left\|(-A)^{\alpha} \int_{0}^{s} e_{2}(s, \tau, u(\tau)) d \tau\right\| d s, \\
Q_{3} & =C_{\alpha} \int_{0}^{t} \frac{\|f(\cdot, 0,0)\|}{(t-s)^{\alpha}} d s .
\end{aligned}
$$

Again by using Lemma 2.1 and 2.2 of [14], we have

$$
\begin{aligned}
Q_{1} & =C_{\alpha} \int_{0}^{t} \frac{K_{f}(s)}{(t-s)^{\alpha}}\left\|(-A)^{\alpha} u(s)\right\| d s \\
\leq & C_{\alpha} \int_{0}^{t} \frac{K_{f}(s)}{(t-s)^{\alpha}} \frac{\|u\|_{\alpha}}{\left(s-t_{i}\right)^{\alpha}} d s \text { for } s \in\left(t_{i}, t_{i+1}\right], \text { and } i=\{1, \ldots, N\} \\
\leq & C_{\alpha} \frac{\left\|K_{f}\right\|_{L^{q^{\prime}}\left(\left[0, t_{j}\right]\right)}}{\left(t-t_{j}\right)^{\alpha}}\left(N+\frac{2^{\alpha+\frac{1}{q}}}{(1-\alpha q)^{\frac{1}{q}}}\right)\left[\int_{0}^{a}\left(\frac{2^{\alpha q}\|u\|_{\alpha}^{q}}{s^{\alpha q}}\right) d s\right]^{\frac{1}{q}} \\
& \quad \text { for } t \in\left(t_{j}, t_{j+1}\right], j=\{1, \ldots, N\}
\end{aligned}
$$




$$
\begin{aligned}
& \text { where } N=\left(\sum_{i=0}^{j-1} \int_{0}^{t_{i+1}-t_{i}}\left(\frac{\|u\|_{\alpha}}{s^{\alpha}}\right) d s\right)^{\frac{1}{q}} \text {. } \\
& Q_{2}=C_{\alpha} \int_{0}^{t} \frac{\widetilde{K}_{f}(s)}{(t-s)^{\alpha}}\left\|(-A)^{\alpha} \int_{0}^{s} e_{2}(s, \tau, u(\tau)) d \tau\right\| d s \\
& \leq C_{\alpha} \int_{0}^{t} \frac{\widetilde{K}_{f}(s)}{(t-s)^{\alpha}}\left\|(-A)^{\alpha}\right\| \int_{0}^{s} K_{e_{2}}(s, \tau)\|u(\tau)\| d \tau d s \\
& \leq C_{\alpha} \int_{0}^{t} \frac{\widetilde{K}_{f}(s) K_{e_{2}}^{*}}{(t-s)^{\alpha}}\left\|(-A)^{\alpha} u(s)\right\| d s \\
& \leq C_{\alpha} K_{e_{2}}^{*} \int_{0}^{t} \frac{\widetilde{K}_{f}(s)}{(t-s)^{\alpha}} \frac{\|u\|_{\alpha}}{\left(s-t_{i}\right)^{\alpha}} d s \\
& \text { for } s \in\left(t_{i}, t_{i+1}\right] \text {, and } i=\{1, \ldots, N\} \\
& \leq C_{\alpha} K_{e_{2}}^{*} \frac{\left\|\widetilde{K}_{f}\right\|_{L^{q^{\prime}}\left(\left[0, t_{j}\right]\right)}}{\left(t-t_{j}\right)^{\alpha}}\left(N+\frac{2^{\alpha+\frac{1}{q}}}{(1-\alpha q)^{\frac{1}{q}}}\right)\left[\int_{0}^{a}\left(\frac{2^{\alpha q}\|u\|_{\alpha}^{q}}{s^{\alpha q}}\right) d s\right]^{\frac{1}{q}} \\
& \text { for } t \in\left(t_{j}, t_{j+1}\right], j=\{1, \ldots, N\} \\
& Q_{3}=\frac{C_{\alpha}}{(t-s)^{\alpha}} \int_{0}^{t}\|f(\cdot, 0,0)\| d s \\
& =C_{\alpha} \int_{0}^{t}\|f(\cdot, 0,0)\|(t-s)^{-(\alpha)} d s \\
& =C_{\alpha} \frac{\left(t-t_{j}\right)^{\alpha}}{\left(t-t_{j}\right)^{\alpha}} \frac{t^{(1-\alpha)}}{(1-\alpha)}\|f(\cdot, 0,0)\|_{L^{1}([0, a])} \\
& =\frac{C_{\alpha} p_{3} K_{f}^{*}}{\left(t-t_{j}\right)^{\alpha}}
\end{aligned}
$$

where $K_{f}^{*}=\|f(\cdot, 0,0)\|_{L^{1}([0, a])}$ and $p_{3}=\frac{\left(t-t_{j}\right)^{\alpha} t^{(1-\alpha)}}{(1-\alpha)}$.

Hence by combining the estimates $\left(Q_{1}\right)-\left(Q_{3}\right)$ and together with (3.5), we obtain

$$
\begin{aligned}
J_{3} \leq & C_{\alpha} \frac{\left\|K_{f}\right\|_{L^{q^{\prime}}\left(\left[0, t_{j}\right]\right)}}{\left(t-t_{j}\right)^{\alpha}}\left(N+\frac{2^{\alpha+\frac{1}{q}}}{(1-\alpha q)^{\frac{1}{q}}}\right)\left[\int_{0}^{a}\left(\frac{2^{\alpha q}\|u\|_{\alpha}^{q}}{s^{\alpha q}}\right) d s\right]^{\frac{1}{q}} \\
& +C_{\alpha} K_{e_{2}}^{*} \frac{\left\|\widetilde{K}_{f}\right\|_{L^{q^{\prime}}\left(\left[0, t_{j}\right]\right)}}{\left(t-t_{j}\right)^{\alpha}}\left(N+\frac{2^{\alpha+\frac{1}{q}}}{(1-\alpha q)^{\frac{1}{q}}}\right)\left[\int_{0}^{a}\left(\frac{2^{\alpha q}\|u\|_{\alpha}^{q}}{s^{\alpha q}}\right) d s\right]^{\frac{1}{q}} \\
& +\frac{C_{\alpha} p_{3} K_{f}^{*}}{\left(t-t_{j}\right)^{\alpha}}
\end{aligned}
$$


EXISTENCE RESULTS FOR AN IMPULSIVE NEUTRAL

$$
\begin{aligned}
\leq & \frac{C_{\alpha}}{\left(t-t_{j}\right)^{\alpha}}\left\{\left(\left\|K_{f}\right\|_{L^{q^{\prime}}\left(\left[0, t_{j}\right]\right)}+K_{e_{2}}^{*}\left\|\widetilde{K}_{f}\right\|_{L^{q^{\prime}}\left(\left[0, t_{j}\right]\right)}\right)\right. \\
& \left.\left(N+\frac{2^{\alpha+\frac{1}{q}}}{(1-\alpha q)^{\frac{1}{q}}}\right)\left[\int_{0}^{a}\left(\frac{2^{\alpha q}\|u\|_{\alpha}^{q}}{s^{\alpha q}}\right) d s\right]^{\frac{1}{q}}+p_{3} K_{f}^{*}\right\} \\
\leq & \frac{C_{\alpha}}{\left(t-t_{j}\right)^{\alpha}}\left\{\left(\left\|K_{f}\right\|_{L^{q^{\prime}}\left(\left[0, t_{j}\right]\right)}+K_{e_{2}}^{*}\left\|\widetilde{K}_{f}\right\|_{L^{q^{\prime}}\left(\left[0, t_{j}\right]\right)}\right)\right. \\
& \left.\left(N+\frac{2^{\alpha+\frac{1}{q}}}{(1-\alpha q)^{\frac{1}{q}}}\right)\left[\frac{2^{\alpha} a^{1 / q-\alpha}}{(1-q \alpha)^{1 / q}}\right]\|u\|_{\alpha}+p_{3} K_{f}^{*}\right\} \\
\leq & \frac{C_{\alpha}}{\left(t-t_{j}\right)^{\alpha}}\left\{\Upsilon\|u\|_{\alpha}+p_{3} K_{f}^{*}\right\},
\end{aligned}
$$

where $\Upsilon=\left\{\left(\left\|K_{f}\right\|_{L^{q^{\prime}}\left(\left[0, t_{j}\right]\right)}+K_{e_{2}}^{*}\left\|\widetilde{K}_{f}\right\|_{L^{q^{\prime}}\left(\left[0, t_{j}\right]\right)}\right)\right.$

$$
\left.\times\left(N+\frac{2^{\alpha+\frac{1}{q}}}{(1-\alpha q)^{\frac{1}{q}}}\right)\left[\frac{2^{\alpha} a^{1 / q-\alpha}}{(1-q \alpha)^{1 / q}}\right]\right\} .
$$

Finally, Let $J_{4}=\left\|(-A)^{\alpha} \sum_{i=1}^{j} T\left(t-t_{i}\right) I_{i}\left(u\left(t_{i}\right)\right)\right\|$, for $j=1, \ldots, N$.

$$
\begin{aligned}
& \leq \sum_{i=1}^{j}\left\|(-A)^{\alpha} T\left(t-t_{i}\right)\right\|\left\|I_{i}\left(u\left(t_{i}\right)\right)\right\| \\
& \leq \frac{C_{\alpha}}{\left(t-t_{j}\right)^{\alpha}} \sum_{i=1}^{j}\left(\left\|I_{i}\left(u\left(t_{i}\right)\right)-I_{i}(0)\right\|+\left\|I_{i}(0)\right\|\right) \\
& \leq \frac{C_{\alpha}}{\left(t-t_{j}\right)^{\alpha}} \sum_{i=1}^{j}\left(K_{I_{i}}(l)\left\|(-A)^{\alpha} u\right\|+\left\|I_{i}(0)\right\|\right) \\
& \leq \frac{C_{\alpha}}{\left(t-t_{j}\right)^{\alpha}} \sum_{i=1}^{j}\left(K_{I_{i}}\left(\frac{r}{\delta_{i-1}^{\alpha}}\right) \frac{\|u\|_{\alpha}^{q}}{\delta_{i-1}^{\alpha}}+\left\|I_{i}(0)\right\|\right)
\end{aligned}
$$

For $t \in\left(t_{j}, t_{j+1}\right]$, by combining the above results in (3.4), we have

$$
\begin{aligned}
\left\|(-A)^{\alpha} \Gamma u(t)\right\|= & \|(-A)^{\alpha} T(t)\left[u_{0}-g\left(0, u_{0}, 0\right)\right] \\
& +(-A)^{\alpha} g\left(t, u(t), \int_{0}^{t} e_{1}(t, s, u(s)) d s\right) \\
& +(-A)^{\alpha} \int_{0}^{t} T(t-s) f\left(s, u(s), \int_{0}^{s} e_{2}(s, \tau, u(\tau)) d \tau\right) d s
\end{aligned}
$$




$$
\begin{aligned}
\leq & \frac{C_{\alpha}}{\left(t-t_{j}\right)^{\alpha}}\left[\left\|u_{0}\right\|+\left\|g\left(0, u_{0}, 0\right)\right\|\right] \\
& +\frac{C_{\alpha}}{\left(t-t_{j}\right)^{\alpha}}\left\{\Upsilon\|u\|_{\alpha}+p_{3} K_{f}^{*}\right\} \\
& +\frac{C_{\alpha}}{\left(t-t_{j}\right)^{\alpha}} \sum_{i=1}^{j}\left[K_{I_{i}}\left(\frac{r}{\delta_{i-1}^{\alpha}}\right) \frac{\|u\|_{\alpha}}{\delta_{i-1}^{\alpha}}+\left\|I_{i}(0)\right\|\right] \\
\leq & \frac{C_{\alpha}}{\left(t-t_{j}\right)^{\alpha}}\left[\left\|u_{0}\right\|+\left\|g\left(0, u_{0}, 0\right)\right\|+p_{2}+p_{3} K_{f}^{*}+\sum_{i=1}^{j}\left\|I_{i}(0)\right\|\right] \\
& +\frac{C_{\alpha}\|u\|_{\alpha}}{\left(t-t_{j}\right)^{\alpha}}\left\{p_{1}+\Upsilon+\sum_{i=1}^{j} \frac{K_{I_{i}}\left(\frac{r}{\delta_{i-1}^{\alpha}}\right)}{\delta_{i-1}^{\alpha}}\right\}
\end{aligned}
$$

So that, we have for $t \in\left(t_{j}, t_{j+1}\right]$,

$$
\begin{array}{r}
\left\|\Gamma_{n} u\right\|_{\alpha, j} \leq C_{\alpha}\left[\left\|u_{0}\right\|+\|\right. \\
\left.+\left(0, u_{0}, 0\right)\left\|+p_{2}+p_{3} K_{f}^{*}+\sum_{i=1}^{j}\right\| I_{i}(0) \|\right] \\
+C_{\alpha}\|u\|_{\alpha}\left\{p_{1}+\Upsilon+\sum_{i=1}^{j} \frac{K_{I_{i}}\left(\frac{r}{\delta_{i-1}^{\alpha}}\right)}{\delta_{i-1}^{\alpha}}\right\}
\end{array}
$$

which implies that

$$
\|\Gamma u\|_{\alpha} \leq C_{\alpha}\left\|u_{0}\right\|+C_{\alpha}\left[\left\|g\left(0, u_{0}, 0\right)\right\|+p_{2}+p_{3} K_{f}^{*}+\sum_{i=1}^{j}\left\|I_{i}(0)\right\|\right]+\Theta r \leq r
$$

and $\Gamma u \in B_{r}\left(0, P C_{\alpha}\left(X_{\alpha}\right)\right)$. Then for $u, v \in B_{r}\left(0, P C_{\alpha}\left(X_{\alpha}\right)\right)$, we have

$$
\|\Gamma u-\Gamma v\|_{\alpha, j} \leq C_{\alpha}\left\{p_{1}+\Upsilon+\sum_{i=1}^{j} \frac{K_{I_{i}}\left(\frac{r}{\delta_{i-1}^{\alpha}}\right)}{\delta_{i-1}^{\alpha}}\right\}\|u-v\|_{\alpha}
$$

which shows that

$$
\|\Gamma u-\Gamma v\|_{\alpha} \leq \Theta\|u-v\|_{\alpha},
$$

for all $u, v \in B_{r}\left(0, P C_{\alpha}\left(X_{\alpha}\right)\right)$ and $\Gamma$ is a contraction on $P C_{\alpha}\left(X_{\alpha}\right)$ when

$$
\Theta=C_{\alpha}\left\{p_{1}+\Upsilon+\sum_{i=1}^{j} \frac{K_{I_{i}}\left(\frac{r}{\delta_{i-1}^{\alpha}}\right)}{\delta_{i-1}^{\alpha}}\right\}<1
$$

Thus there exists a unique fixed point $\mathrm{v} \in B_{r}\left(0, P C_{\alpha}\left(X_{\alpha}\right)\right)$ of $\Gamma(\cdot)$. Next we prove that $u(\cdot)$ is a mild solution of the system $(2.1)-(2.3)$. Obviously all the limits $u\left(t_{i}^{+}\right)$exist when $u(\cdot)$ is left continuous on $J$. 
For that we need the following results. For all $t \in\left(t_{j}, t_{j+1}\right]$ by the hypothesis $(H 1)-(H 4)$ and Hölder's inequality, we have

$$
\begin{aligned}
J_{5}= & \left\|g\left(t, u(t), \int_{0}^{t} e_{1}(t, s, u(s)) d s\right)\right\| \\
\leq & K_{g}(t)\left\|(-A)^{\alpha} u(t)\right\|+\widetilde{K}_{g}(t)\left\|(-A)^{\alpha} \int_{0}^{t} e_{1}(t, s, u(s)) d s\right\| \\
& \quad+\|g(\cdot, 0,0)\| \\
\leq & K_{g}(t)\left\|(-A)^{\alpha} u(t)\right\|+\widetilde{K}_{g}(t) K_{e_{1}}^{*}\left\|(-A)^{\alpha} u(t)\right\|+\|g(\cdot, 0,0)\| \\
\leq & K_{g}(t) \frac{\|u\|_{\alpha}}{\left(t-t_{i}\right)^{\alpha}}+\widetilde{K}_{g}(t) K_{e_{1}}^{*} \frac{\|u\|_{\alpha}}{\left(t-t_{i}\right)^{\alpha}}+K_{g}^{*} \cdot \\
J_{6}= & \left\|\int_{0}^{t} T(t-s) f\left(s, u(s), \int_{0}^{s} e_{2}(s, \tau, u(\tau)) d \tau\right) d s\right\| \\
\leq & \left\|\sum_{i=0}^{j-1} \int_{t_{i}}^{t_{i+1}} T(t-s) f\left(s, u(s), \int_{0}^{s} e_{2}(s, \tau, u(\tau)) d \tau\right) d s\right\| \\
& \quad\left\|\int_{t_{j}}^{t} T(t-s) f\left(s, u(s), \int_{0}^{s} e_{2}(s, \tau, u(\tau)) d \tau\right) d s\right\| .
\end{aligned}
$$

For further simplification, let $J_{6}=Q_{4}+Q_{5}$,

$$
\begin{aligned}
\text { where } Q_{4}= & \left\|\sum_{i=0}^{j-1} \int_{t_{i}}^{t_{i+1}} T(t-s) f\left(s, u(s), \int_{0}^{s} e_{2}(s, \tau, u(\tau)) d \tau\right) d s\right\| \\
\text { and } Q_{5}= & \left\|\int_{t_{j}}^{t} T(t-s) f\left(s, u(s), \int_{0}^{s} e_{2}(s, \tau, u(\tau)) d \tau\right) d s\right\| . \\
Q_{4}= & \left\|\sum_{i=0}^{j-1} \int_{t_{i}}^{t_{i+1}} T(t-s) f\left(s, u(s), \int_{0}^{s} e_{2}(s, \tau, u(\tau)) d \tau\right) d s\right\| \\
\leq & \sum_{i=0}^{j-1} C_{0} \int_{t_{i}}^{t_{i+1}}\left[K_{f}(s)\left\|(-A)^{\alpha} u(s)\right\|\right. \\
& \left.\left.+\widetilde{K}_{f}(s) \|(-A)^{\alpha} \int_{0}^{s} e_{2}(s, \tau, u(\tau)) d \tau\right)\|+\| f(\cdot, 0,0) \|\right] d s \\
\leq & \sum_{i=0}^{j-1} C_{0} \int_{t_{i}}^{t_{i+1}}\left[K_{f}(s) \frac{\|u\|_{\alpha}}{\left(s-t_{i}\right)^{\alpha}}+K_{e_{2}}^{*} \widetilde{K}_{f}(s) \frac{\|u\|_{\alpha}}{\left(s-t_{i}\right)^{\alpha}}\right. \\
& +\|f(\cdot, 0,0)\|] d s
\end{aligned}
$$


EXISTENCE RESULTS FOR AN IMPULSIVE NEUTRAL

$$
\begin{aligned}
\leq & C_{0}\left[\left\|K_{f}\right\|_{L^{q^{\prime}([0, a])}}+\left\|\widetilde{K}_{f}\right\|_{L^{q^{\prime}([0, a])}} K_{e_{2}}^{*}\right] \\
& \times\left(\sum_{i=0}^{j-1} \int_{0}^{t_{i+1}-t_{i}}\left(\frac{\|u\|_{\alpha}}{s^{\alpha}}\right) d s\right)^{\frac{1}{q}} \\
& +\sum_{i=0}^{j-1} C_{0} \int_{t_{i}}^{t_{i+1}}\|f(\cdot, 0,0)\| d s \\
\leq & C_{0}\left[\left\|K_{f}\right\|_{L^{q^{\prime}([0, a])}}+\left\|\widetilde{K}_{f}\right\|_{L^{q^{\prime}}([0, a])} K_{e_{2}}^{*}\right] N+C_{0}\|f(\cdot, 0,0)\|_{L^{1}([0, a])} \\
\leq & C_{0}\left[\left\|K_{f}\right\|_{L^{q^{\prime}}([0, a])}+\left\|\widetilde{K}_{f}\right\|_{L^{q^{\prime}([0, a])}} K_{e_{2}}^{*}\right] N+C_{0} K_{f}^{*},
\end{aligned}
$$

where $N=\left(\sum_{i=0}^{j-1} \int_{0}^{t_{i+1}-t_{i}}\left(\frac{\|u\|_{\alpha}}{s^{\alpha}}\right) d s\right)^{\frac{1}{q}}$.

Similarly by using above discussions for $Q_{5}$, we have,

$$
\begin{aligned}
Q_{5}= & \left\|\int_{t_{j}}^{t} T(t-s) f\left(s, u(s), \int_{0}^{s} e_{2}(s, \tau, u(\tau)) d \tau\right) d s\right\| \\
\leq & C_{0} \int_{t_{j}}^{t}\left[K_{f}(s)\left\|(-A)^{\alpha} u(s)\right\|+\widetilde{K}_{f}(s) \|(-A)^{\alpha} \int_{0}^{s} e_{2}(s, \tau, u(\tau)) d \tau\right) \| \\
& +\|f(\cdot, 0,0)\|] d s \\
\leq & C_{0} \int_{t_{j}}^{t}\left[K_{f}(s) \frac{\|u\|_{\alpha}}{\left(s-t_{i}\right)^{\alpha}}+K_{e_{2}}^{*} \widetilde{K}_{f}(s) \frac{\|u\|_{\alpha}}{\left(s-t_{i}\right)^{\alpha}}+\|f(\cdot, 0,0)\|\right] d s \\
\leq & C_{0}\left[\left\|K_{f}\right\|_{L^{q^{\prime}}([0, a])}+\left\|\widetilde{K}_{f}\right\|_{L^{q^{\prime}}([0, a])} K_{e_{2}}^{*}\right] r \frac{a^{\frac{1}{q}-\alpha}}{(1-\alpha q)^{\frac{1}{q}}} \\
& +C_{0}\|f(\cdot, 0,0)\|_{L^{1}([0, a])}
\end{aligned}
$$

Hence, $J_{6} \leq C_{0}\left[\left\|K_{f}\right\|_{L^{q^{\prime}}([0, a])}+\left\|\widetilde{K}_{f}\right\|_{L^{q^{\prime}([0, a])}} K_{e_{2}}^{*}\right] N+C_{0} K_{f}^{*}$

$$
\begin{aligned}
& +C_{0}\left[\left\|K_{f}\right\|_{L^{q^{\prime}([0, a])}}+\left\|\widetilde{K}_{f}\right\|_{L^{q^{\prime}([0, a])}} K_{e_{2}}^{*}\right] r \frac{a^{\frac{1}{q}-\alpha}}{(1-\alpha q)^{\frac{1}{q}}}+C_{0} K_{f}^{*} \\
J_{6} \leq & C_{0}\left[\left\|K_{f}\right\|_{L^{q^{\prime}([0, a])}}+\left\|\widetilde{K}_{f}\right\|_{L^{q^{\prime}([0, a])}} K_{e_{2}}^{*}\right] \\
& \times\left(N+r \frac{a^{\frac{1}{q}-\alpha}}{(1-\alpha q)^{\frac{1}{q}}}\right)+2 C_{0} K_{f}^{*} .
\end{aligned}
$$

By utilizing above results and from (3.3) and for $t \in\left(t_{j}, t_{j+1}\right]$, we have 


$$
\begin{aligned}
\|u(t)\| \leq & C_{0}\left\|u_{0}\right\|+C_{0}\left\|g\left(0, u_{0}, 0\right)\right\|+\left\|g\left(t, u(t), \int_{0}^{t} e_{1}(t, s, u(s)) d s\right)\right\| \\
& +\left\|\sum_{i=0}^{j-1} \int_{t_{i}}^{t_{i+1}} T(t-s) f\left(s, u(s), \int_{0}^{s} e_{2}(s, \tau, u(\tau)) d \tau\right) d s\right\| \\
& +\left\|\int_{t_{j}}^{t} T(t-s) f\left(s, u(s), \int_{0}^{s} e_{2}(s, \tau, u(\tau)) d \tau\right) d s\right\| \\
& +C_{0} \sum_{i=1}^{j}\left(K_{I_{i}}\left(\frac{r}{\delta_{i-1}^{\alpha}}\right) \frac{\|u\|_{\alpha}}{\delta_{i-1}^{\alpha}}+\left\|I_{i}(0)\right\|\right) .
\end{aligned}
$$

By using $J_{5}$ and $J_{6}$, we have

$$
\begin{aligned}
\|u(t)\| \leq & C_{0}\left\|u_{0}\right\|+C_{0}\left\|g\left(0, u_{0}, 0\right)\right\|+K_{g}(t) \frac{\|u\|_{\alpha}}{\left(t-t_{i}\right)^{\alpha}} \\
& +\widetilde{K}_{g}(t) K_{e_{1}}^{*} \frac{\|u\|_{\alpha}}{\left(t-t_{i}\right)^{\alpha}}+K_{g}^{*}(t)+C_{0}\left[\left\|K_{f}\right\|_{L^{q^{\prime}}([0, a])}\right. \\
& \left.+\left\|\widetilde{K}_{f}\right\|_{L^{q^{\prime}([0, a])}} K_{e_{2}}^{*}\right]\left(N+r \frac{a^{\frac{1}{q}-\alpha}}{(1-\alpha q)^{\frac{1}{q}}}\right) \\
& +2 C_{0} K_{f}^{*}+C_{0} \sum_{i=1}^{j}\left(K_{I_{i}}\left(\frac{r}{\delta_{i-1}^{\alpha}}\right) \frac{\|u\|_{\alpha}}{\delta_{i-1}^{\alpha}}+\left\|I_{i}(0)\right\|\right) \\
< & \infty
\end{aligned}
$$

which gives that $\|u\|_{P C(X)}<\infty$. Therefore $\|\Gamma u\|_{P C(X)}$ is finite. Hence $u(\cdot) \in J$ is a mild solution of the problem $(2.1)-(2.3)$.

Corollary 3.1. If the conditions $\left(H_{1}\right)-\left(H_{4}\right)$ hold and

$$
\Theta=C_{\alpha}\left\{p_{1}+\Upsilon+\sum_{i=1}^{j} \frac{K_{I_{i}}\left(\frac{r}{\delta_{i-1}^{\alpha}}\right)}{\delta_{i-1}^{\alpha}}\right\}<1 .
$$

then there exists a unique mild solution $u \in P C_{\alpha}\left(X_{\alpha}\right)$ of (2.1)-(2.3). If the functions $K_{g}, \widetilde{K}_{g}, K_{f}, \widetilde{K}_{f}, K_{I_{i}}$ are bounded and $C_{\alpha}\left\{p_{1}+\Upsilon\right\}<1$ where

$$
\begin{array}{r}
\Upsilon=\left\{\left(\left\|K_{f}\right\|_{L^{q^{\prime}}\left(\left[0, t_{j}\right]\right)}+K_{e_{2}}^{*}\left\|\widetilde{K}_{f}\right\|_{L^{q^{\prime}}\left(\left[0, t_{j}\right]\right)}\right)\right. \\
\left.\left(N+\frac{2^{\alpha+\frac{1}{q}}}{(1-\alpha q)^{\frac{1}{q}}}\right)\left[\frac{2^{\alpha} a^{1 / q-\alpha}}{(1-q \alpha)^{1 / q}}\right]\right\}
\end{array}
$$


and

$$
p_{1}=\left[M_{0} K_{g}(t) \frac{\left(t-t_{j}\right)^{\alpha}}{C_{\alpha}\left(t-t_{i}\right)^{\alpha}}+M_{0} \widetilde{K}_{g}(t) K_{e_{1}}^{*} \frac{\left(t-t_{j}\right)^{\alpha}}{C_{\alpha}\left(t-t_{i}\right)^{\alpha}}\right]
$$

then there exists a unique mild solution $u \in P C_{\alpha}\left(X_{\alpha}\right)$ of $(2.1)-(2.3)$.

Proof. Assume $C_{\alpha}\left\{p_{1}+\Upsilon+\sum_{i=1}^{j} \frac{K_{I_{i}}\left(\frac{r}{\delta_{i-1}^{\alpha}}\right)}{\delta_{i-1}^{\alpha}}\right\} \leq \Theta r$ for $r>0, \Theta \in(0,1)$ and $\Omega$ defined in (3.2) where $\Omega<(1-\Theta) r$ and $\left\|u_{0}\right\| \leq \frac{((1-\Theta) r-\Omega)}{C_{\alpha}}$.

Now, the assertion follows from the Theorem (3.1).

The main results of this section, the Theorem (3.1), establishes the conditions for the existence of a mild solution via the Krasnoselskii-Schaefers fixed point theorem. We divide the verification into various steps. We define $\Gamma^{k} B_{r}\left(0, P C_{\alpha}\left(X_{\alpha}\right)\right)(s)=\left\{\Gamma^{k} u(s): u \in B_{r}\left(0, P C_{\alpha}\left(X_{\alpha}\right)\right)\right\}$, where $k=1,2$. For $s, t \in\left(t_{i}, t_{i+1}\right]$, we have

(H6) For $x \in X$, the function $f(\cdot, \varphi, \mu)$ is strongly measurable on $[0, a]$ and $f(t, \varphi, \mu) \in C\left(J \times X_{\alpha} \times X_{\alpha} ; X\right)$ for each $t \in J$. There are $q \in\left[1, \frac{1}{\alpha}\right)$, $m_{f} \in L^{q^{\prime}}\left([0, a] ; R^{+}\right)$and there exist a function $W_{f} \in C\left([0, \infty) ; R^{+}\right)$ which is non decreasing such that

$$
\|f(t, \varphi, \mu)\| \leq m_{f}(t) W_{f}\left(\left\|(-A)^{\alpha} \varphi\right\|+\left\|(-A)^{\alpha} \mu\right\|\right),
$$

for all $t \in J$ and $\varphi, \mu \in P C_{\alpha}\left(X_{\alpha}\right)$.

(H7) The function $I_{i} \in C\left(P C_{\alpha}\left(X_{\alpha}\right) ; X\right)$ and there are non decreasing functions $c_{i}:[0, \infty) \rightarrow \mathbb{R}^{+}$and the constants $d_{i}>0$ such that

$$
\left\|I_{i}(x)\right\| \leq c_{i}(l)\left\|(-A)^{\alpha} x\right\|+d_{i},
$$

for $l>0$ and for each $i=1,2, \ldots, N$.

Now we decompose $\Gamma_{n}$ as $\Gamma^{1}+\Gamma^{2}$ where

$$
\begin{aligned}
\Gamma^{1}(u(t))= & T(t)\left[u_{0}+g\left(0, u_{0}, 0\right)\right]-g\left(t, u(t), \int_{0}^{t} e_{1}(t, s, u(s)) d s\right) \\
\Gamma^{2}(u(t))= & \int_{0}^{t} T(t-s) f\left(s, u(s), \int_{0}^{s} e_{2}(s, \tau, u(\tau)) d \tau\right) d s \\
& +\sum_{t_{i}<t} T\left(t-t_{i}\right) I_{i}\left(u\left(t_{i}\right)\right), \quad \text { for all } t \in[0, a] .
\end{aligned}
$$

Theorem 3.2. If the assumptions (H5)-(H6) hold, then (2.1)-(2.3) admits at least one mild solution on J. 
EXISTENCE RESULTS FOR AN IMPULSIVE NEUTRAL

Proof. First we have to prove that the operator $\Gamma_{n}$ is completely continuous. We divide the verification into various steps and we utilize the script $\Gamma^{i} B_{r}\left(0, P C_{\alpha}\left(X_{\alpha}\right)\right)=\left\{\Gamma^{i} u(s): u \in B_{r}\left(0, P C_{\alpha}\left(X_{\alpha}\right)\right)\right\}$.

Step 1.

By Theorem 3.1 we conclude that $\Gamma^{1}$ is a contraction on $B_{r}\left(0, P C_{\alpha}\left(X_{\alpha}\right)\right)$. Indeed $u, v \in B_{r}\left(0, P C_{\alpha}\left(X_{\alpha}\right)\right)$, we have

$$
\begin{aligned}
\left\|\Gamma^{1}(u(t))-\Gamma^{1}(v(t))\right\| & \leq\left\{\frac{K_{g}(t)}{\left(t-t_{i}\right)^{\alpha}}+\frac{\widetilde{K}_{g}(t) K_{e_{1}}^{*}}{\left(t-t_{i}\right)^{\alpha}}\right\}\|u-v\|_{\alpha} \\
& \leq C_{0}\|u-v\|_{\alpha} .
\end{aligned}
$$

Since $C_{0}=\left\{\frac{K_{g}(t)}{\left(t-t_{i}\right)^{\alpha}}+\frac{\widetilde{K}_{g}(t) K_{e_{1}}^{*}}{\left(t-t_{i}\right)^{\alpha}}\right\}<1, \Gamma^{1}$ is a contraction on $B_{r}\left(0, P C_{\alpha}\left(X_{\alpha}\right)\right)$.

\section{Step 2.}

Next we prove that the operator $\Gamma^{2}$ is completely continuous. First, we have to prove that $\Gamma^{2}$ maps bounded sets into bounded sets in $B_{r}\left(0, P C_{\alpha}\left(X_{\alpha}\right)\right)$. Let us consider

$$
\Gamma^{2} B_{r}\left(0, P C_{\alpha}\left(X_{\alpha}\right)\right)=\left\{\Gamma^{2} u: u \in B_{r}\left(0, P C_{\alpha}\left(X_{\alpha}\right)\right)\right\} .
$$

It is suffice to show that there exist a positive constant $\xi$ such that for each $u \in B_{r}\left(0, P C_{\alpha}\left(X_{\alpha}\right)\right)$, we have $\left\|\Gamma^{2} u\right\| \leq \xi$.

Now for $t \in\left(t_{j}, t_{j+1}\right]$,

$$
\begin{aligned}
\left\|(-A)^{\alpha} \Gamma^{2}(u(t))\right\|= & \|(-A)^{\alpha} \int_{0}^{t} T(t-s) f\left(s, u(s), \int_{0}^{s} e_{2}(s, \tau, u(\tau)) d \tau\right) d s \\
\leq & \frac{C_{\alpha}}{\left(t-t_{j}\right)^{\alpha}} \int_{0}^{t} \frac{m_{f}(s)}{(t-s)^{\alpha}} W_{f}\left\{\left(\frac{\|u(s)\|_{\alpha}}{\left(s-t_{i}\right)^{\alpha}}\right)\right. \\
& \left.+K_{e_{2}}^{*}\left(\frac{\|u(s)\|_{\alpha}}{\left(s-t_{i}\right)^{\alpha}}\right)\right\} d s \\
& +\frac{C_{\alpha}}{\left(t-t_{j}\right)^{\alpha}} \sum_{i=1}^{j}\left[c_{i}\left(\frac{r}{\delta_{i-1}^{\alpha}}\right) \frac{\|u\|_{\alpha}}{\delta_{i-1}^{\alpha}}+d_{i}\right] \\
\leq & C_{\alpha}\left(1+K_{e_{2}}^{*}\right) \frac{\left\|m_{f}\right\|_{L^{\prime}([0, a])}}{\left(t-t_{j}\right)^{\alpha}}\left(N+\frac{2^{\alpha+\frac{1}{q}}}{(1-\alpha q)^{\frac{1}{q}}}\right) \\
& \times\left[\int_{0}^{a} W_{f}\left(\frac{2^{\alpha q}\|u\|_{\alpha}^{q}}{s^{\alpha q}}\right)^{\frac{1}{q}}\right]^{\frac{1}{q}} \\
& +\frac{C_{\alpha}}{\left(t-t_{j}\right)^{\alpha}} \sum_{i=1}^{j}\left[c_{i}\left(\frac{r}{\delta_{i-1}^{\alpha}}\right) \frac{\|u\|_{\alpha}}{\delta_{i-1}^{\alpha}}+d_{i}\right] \leq \xi .
\end{aligned}
$$


Also this is true for each $u \in B_{r}\left(0, P C_{\alpha}\left(X_{\alpha}\right)\right)$.

\section{Step 3.}

Now we show that $\Gamma^{2}$ maps $B_{r}$ into a precompact set in $X_{\alpha}$.

That is for $t_{j}<c<d \leq t_{j+1}$ the set $\cup_{s \in[c, d]} \Gamma^{2} B_{r}\left(0, P C_{\alpha}\left(X_{\alpha}\right)\right)(s)$ is relatively compact in $X_{\alpha}$. Let $t_{j}<\varphi<c$ be fixed and $\epsilon$ be a real number satisfying $0<\epsilon<\frac{c-\varphi}{2}$. For $u \in B_{r}\left(0, P C_{\alpha}\left(X_{\alpha}\right)\right)$, we define

$$
\begin{aligned}
\left((-A)^{\alpha} \Gamma_{\epsilon}^{2} u\right)(t) & \\
\leq & \int_{0}^{t-\epsilon}(-A)^{\alpha} T(t-s) f\left(s, u(s), \int_{0}^{s} e_{2}(s, \tau, u(\tau)) d \tau\right) d s \\
& +\sum_{0<t_{i}<t-\epsilon}(-A)^{\alpha} T\left(t-t_{i}\right) I_{i}\left(u\left(t_{i}\right)\right) \\
= & T(\epsilon) \int_{0}^{t-\epsilon}(-A)^{\alpha} T(t-s-\epsilon) f\left(s, u(s), \int_{0}^{s} e_{2}(s, \tau, u(\tau)) d \tau\right) d s \\
& +T(\epsilon) \sum_{0<t_{i}<t-\epsilon}(-A)^{\alpha} T\left(t-t_{i}-\epsilon\right) I_{i}\left(u\left(t_{i}\right)\right)
\end{aligned}
$$

Since $T(\epsilon)$ is a compact operator, the set

$$
V_{\epsilon}(t)=\left\{\left(\Gamma_{\epsilon}^{2} u\right)(t): u \in B_{r}\left(0, P C_{\alpha}\left(X_{\alpha}\right)\right)\right\}
$$

is relatively compact in $X$ for any $\epsilon, 0<\epsilon<t$.

Then for each $u \in \mathscr{B}_{r}$, we have

$$
\begin{aligned}
&\left\|\left((-A)^{\alpha} \Gamma^{2} u\right)(t)-\left((-A)^{\alpha} \Gamma_{\epsilon}^{2} u\right)(t)\right\| \\
& \leq \int_{t-\epsilon}^{t}\left\|(-A)^{\alpha} T(t-s) f\left(s, u(s), \int_{0}^{s} e_{2}(s, \tau, u(\tau)) d \tau\right)\right\| d s \\
& \quad+\sum_{t-\epsilon<t_{i}<t}\left\|(-A)^{\alpha} T\left(t-t_{i}\right) I_{i}\left(u\left(t_{i}\right)\right)\right\| \\
& \leq C_{\alpha} \int_{t-\epsilon}^{t} \frac{m_{f}(s)}{(t-s)^{\alpha}} W_{f}\left\{\left(\frac{\|u(s)\|_{\alpha}}{\left(s-t_{i}\right)^{\alpha}}\right)+K_{e_{2}}^{*}\left(\frac{\|u\|_{\alpha}}{\left(\tau-t_{i}\right)^{\alpha}}\right)\right\} d s \\
&+\frac{C_{\alpha}}{\left(t-t_{i}\right)^{\alpha}} \sum_{t-\epsilon<t_{i}<t}\left[c_{i}\left(\frac{r}{\delta_{i-1}^{\alpha}}\right) \frac{\|u\|_{\alpha}}{\delta_{i-1}^{\alpha}}+d_{i}\right] \\
& \leq C_{\alpha}\left(1+K_{e_{2}}^{*}\right)\left\|m_{f}\right\|_{L^{q^{\prime}}([t-\epsilon, t])} \frac{2^{\alpha+\frac{1}{q}}}{(1-\alpha q)^{\frac{1}{q}}} \frac{1}{\epsilon^{\alpha}}\left[\int_{0}^{\frac{\epsilon}{2}} W_{f}^{q}\left(\frac{2^{\alpha} r}{\tau^{\alpha}}\right) d \tau\right] \\
&+\frac{C_{\alpha}}{\left(t-t_{i}\right)^{\alpha}} \sum_{t-\epsilon<t_{i}<t}\left[c_{i}\left(\frac{r}{\delta_{i-1}^{\alpha}}\right) \frac{\|u\|_{\alpha}}{\delta_{i-1}^{\alpha}}+d_{i}\right] \\
& \leq r_{\epsilon} \rightarrow 0 \text { as } \epsilon \rightarrow 0
\end{aligned}
$$


EXISTENCE RESULTS FOR AN IMPULSIVE NEUTRAL

and there are precompact sets arbitrarily close to the set

$$
V_{\epsilon}(t)=\left\{\left(\Gamma_{\epsilon}^{2}(u)\right)(t): u \in B_{r}\left(0, P C_{\alpha}\left(X_{\alpha}\right)\right)\right\} .
$$

Thus the set $\left\{\left(\Gamma^{2} \epsilon\right)(t): u \in B_{r}\left(0, P C_{\alpha}\left(X_{\alpha}\right)\right)\right\}$ is precompact in $X_{\alpha}$.

Step 4.

Next we prove that $\Gamma^{2} B_{r}\left(0, P C_{\alpha}\left(X_{\alpha}\right)\right)=\left\{\Gamma^{2} u: u \in B_{r}\left(0, P C_{\alpha}\left(X_{\alpha}\right)\right)\right\}$ is equicontinuous. In order to prove the equicontinuity of $\Gamma^{2}$, we need to decompose $\Gamma^{2}$ as $\Gamma_{1}^{2}+\Gamma_{2}^{2}$ where

$$
\begin{aligned}
\Gamma_{1}^{2} & =\int_{0}^{t} T(t-s) f\left(s, u(s), \int_{0}^{s} e_{2}(s, \tau, u(\tau)) d \tau\right) d s \\
\Gamma_{2}^{2} & =\sum_{t_{i}<t} T\left(t-t_{i}\right) I_{i}\left(u\left(t_{i}\right)\right)
\end{aligned}
$$

To start, we suppose that $t \in\left(t_{j}, t_{j+1}\right)$ for $t \in(0, a]$. Whereas $T(\cdot) \in$ $C((0, a], \mathscr{L}(X))$ and $\Gamma^{2} B_{r}\left(0, P C_{\alpha}\left(X_{\alpha}\right)\right)(t)$ is relatively compact in $X$, for a specific $\epsilon>0$ we can choose $0<\delta \leq \min \left\{t_{j+1}-t\right\}$ such that $\|(T(\theta)-I) x\| \leq \epsilon$ for each $x \in \Gamma^{2} u: u \in B_{r}\left(0, P C_{\alpha}\left(X_{\alpha}\right)\right)(t)$ and entirely $0<\theta \leq \delta$. Also, for $0<h<\delta$ and $u \in B_{r}\left(0, P C_{\alpha}\left(X_{\alpha}\right)\right)$, we have

$\left\|(-A)^{\alpha} \Gamma_{1}^{2} u(t+h)-(-A)^{\alpha} \Gamma_{1}^{2} u(t)\right\|$

$$
\begin{aligned}
\leq & \left\|(T(h)-I)(-A)^{\alpha} \Gamma_{1}^{2} u(t)\right\| \\
& +\left\|(-A)^{\alpha} \int_{t}^{t+h} T(t+h-s) f\left(s, u(s), \int_{0}^{s} e_{2}(s, \tau, u(\tau)) d \tau\right) d s\right\| \\
\leq & \left\|(T(h)-I)(-A)^{\alpha} \Gamma_{1}^{2} u(t)\right\|+C_{\alpha} \int_{t}^{t+h} \frac{m_{f}(s)}{(t+h-s)^{\alpha}} W_{f}\left\{\left(\frac{\|u(s)\|_{\alpha}}{(s-t)^{\alpha}}\right)\right. \\
& \left.+K_{e_{2}}^{*}\left(\frac{\|u(s)\|_{\alpha}}{(s-t)^{\alpha}}\right)\right\} d s \\
\leq & \sup \left\{\left\|(T(h)-I)(-A)^{\alpha} \Gamma_{1}^{2} v(t)\right\|: v \in B_{r}\left(0, P C_{\alpha}\left(X_{\alpha}\right)\right)\right\} \\
& +C_{\alpha} \int_{t}^{t+h} \frac{m_{f}(s)}{(t+h-s)^{\alpha}} W_{f}\left\{\left(\frac{\|u(s)\|_{\alpha}}{(s-t)^{\alpha}}\right)+K_{e_{2}}^{*}\left(\frac{\|u(s)\|_{\alpha}}{(s-t)^{\alpha}}\right)\right\} d s \\
\leq & \epsilon+\frac{C_{\alpha} 2^{\alpha+\frac{1}{q}}}{(1-q \alpha)^{\frac{1}{q}}}\left\|m_{f}\right\|_{L^{q^{\prime}}[t, t+h]}\left(1+K_{e_{2}}^{*}\right) \frac{1}{h^{\alpha}}\left[\int_{0}^{\frac{h}{2}} W_{f}^{q}\left(\frac{2^{\alpha} r}{s^{\alpha}}\right) d s\right]^{\frac{1}{q}}
\end{aligned}
$$

This proves that $(-A)^{\alpha} \Gamma_{1}^{2} B_{r}\left(0, P C_{\alpha}\left(X_{\alpha}\right)\right)$ is right equicontinuous at $t$. Let $t_{j}<\eta<c<t$ and $\cup_{s \in[\eta, t]} \Gamma_{1}^{2} B_{r}\left(0, P C_{\alpha}\left(X_{\alpha}\right)\right)(s)$ is relatively compact in $X_{\alpha}$. For $\epsilon>0$ and for each $x \in \cup_{s \in[\eta, t]} \Gamma_{1}^{2} B_{r}\left(0, P C_{\alpha}\left(X_{\alpha}\right)\right)(s)$ there exists $\delta$ for 
$0<\delta<\frac{(c-\eta)}{2}$ such that $\|(T(\theta)-I) x\| \leq \epsilon$ and entire $0<\theta \leq \delta$. We consider $0<h<\delta$ and $u \in B_{r}\left(0, P C_{\alpha}\left(X_{\alpha}\right)\right)$ and we have

$\left\|(-A)^{\alpha} \Gamma_{1}^{2} u(t-h)-(-A)^{\alpha} \Gamma_{1}^{2} u(t)\right\|$

$$
\begin{aligned}
\leq & \left\|(I-T(h))(-A)^{\alpha} \Gamma_{1}^{2} u(t-h)\right\| \\
& +\left\|(-A)^{\alpha} \int_{t-h}^{t} T(t-s) f\left(s, u(s), \int_{0}^{s} e_{2}(s, \tau, u(\tau)) d \tau\right) d s\right\| \\
\leq & \sup \left\{\left\|(I-T(h))(-A)^{\alpha} \Gamma_{1}^{2} v(s)\right\|: s \in[\eta, t], v \in B_{r}\left(0, P C_{\alpha}\left(X_{\alpha}\right)\right)\right\} \\
& +C_{\alpha} \int_{t-h}^{t} \frac{m_{f}(s)}{(t-s)^{\alpha}} W_{f}\left\{\left(\frac{\|u(s)\|_{\alpha}}{(s-(t-h))^{\alpha}}\right)+K_{e_{2}}^{*}\left(\frac{\|u(s)\|_{\alpha}}{(s-(t-h))^{\alpha}}\right)\right\} d s \\
\leq & \epsilon+\frac{C_{\alpha} 2^{\alpha+\frac{1}{q}}}{(1-q \alpha)^{\frac{1}{q}}}\left\|m_{f}\right\|_{L^{q^{\prime}}[t-h, t]}\left(1+K_{e_{2}}^{*}\right) \frac{1}{h^{\alpha}}\left[\int_{0}^{h} W_{f}^{q}\left(\frac{2^{\alpha} r}{s^{\alpha}}\right) d s\right]^{\frac{1}{q}}
\end{aligned}
$$

This proves that $(-A)^{\alpha} \Gamma_{1}^{2} B_{r}\left(0, P C_{\alpha}\left(X_{\alpha}\right)\right)$ is left equicontinuous at $t$. Thus,

$$
\left\{\Gamma_{1}^{2} u: u \in B_{r}\left(0, P C_{\alpha}\left(X_{\alpha}\right)\right)\right\}
$$

is equicontinuous. The set

$$
\Gamma_{2}^{2} B_{r}\left(0, P C_{\alpha}\left(X_{\alpha}\right)\right)=\left\{\Gamma_{2}^{2} u: u \in B_{r}\left(0, P C_{\alpha}\left(X_{\alpha}\right)\right)\right\}
$$

is equicontinuous at $t \neq t_{i}$ and left equicontinuous at $t=t_{i}$ in the norm of $X_{\alpha}$. Suppose $t \in(0, a]$ is trivial. Assume that $t \in\left(t_{j}, t_{j+1}\right)$.

Since $\Gamma_{2}^{2} B_{r}\left(0, P C_{\alpha}\left(X_{\alpha}\right)\right)(t)$ is relatively compact in $X$, for particular $\epsilon>0$ we can choose $0<\delta \leq \min \left\{t_{j+1}-t\right\}$ such that $\|(T(\theta)-I) x\| \leq \epsilon$ for each $x \in \Gamma_{2}^{2} B_{r}\left(0, P C_{\alpha}\left(X_{\alpha}\right)\right)(t)$ and completely for all $0<\theta \leq \delta$. As a result, for $0<h<\delta$ and $u \in B_{r}\left(0, P C_{\alpha}\left(X_{\alpha}\right)\right)$, we get

$$
\begin{aligned}
\|(-A)^{\alpha} \Gamma_{2}^{2} u(t+h) & -(-A)^{\alpha} \Gamma_{2}^{2} u(t) \| \\
& \leq\left\|(T(h)-I)(-A)^{\alpha} \Gamma_{n}^{3} u(t)\right\| \\
& \leq \sup \left\{\left\|(T(h)-I)(-A)^{\alpha} \Gamma_{2}^{2} v(s)\right\|: v \in B_{r}\left(0, P C_{\alpha}\left(X_{\alpha}\right)\right)\right\} \\
& \leq \epsilon
\end{aligned}
$$

Hence $(-A)^{\alpha} \Gamma_{2}^{2} B_{r}\left(0, P C_{\alpha}\left(X_{\alpha}\right)\right)$ is right equicontinuous at $t$.

Next we show for $t \in\left(t_{j}, t_{j+1}\right]$, the left equicontinuity. Let $t_{j}<\eta<c<t$. Taking into account that $\cup_{s \in[\eta, t]} \Gamma_{2}^{2} B_{r}\left(0, P C_{\alpha}\left(X_{\alpha}\right)\right)(s)$ is relatively compact in $X_{\alpha}$, for $\epsilon>0$ there exists $0<\delta<\frac{(c-\eta)}{2}$ such that $\|(T(\theta)-I) x\| \leq \epsilon$ for every $x \in \cup_{s \in[\eta, t]} \Gamma_{2}^{2} B_{r}\left(0, P C_{\alpha}\left(X_{\alpha}\right)\right)(s)$ and all $0<\theta \leq \delta$. For $0<h<\delta$ and $u \in B_{r}\left(0, P C_{\alpha}\left(X_{\alpha}\right)\right)$, we have 
EXISTENCE RESULTS FOR AN IMPULSIVE NEUTRAL

$$
\begin{array}{rl}
\|(-A)^{\alpha} \Gamma_{2}^{2} & u(t-h)-(-A)^{\alpha} \Gamma_{2}^{2} u(t) \| \\
& \leq\left\|(I-T(h))(-A)^{\alpha} \Gamma_{2}^{2} u(t-h)\right\| \\
& \leq \sup \left\{\left\|(I-T(h))(-A)^{\alpha} \Gamma_{2}^{2} v(s)\right\|: v \in B_{r}\left(0, P C_{\alpha}\left(X_{\alpha}\right)\right)\right\} \\
& \leq \epsilon
\end{array}
$$

which shows that $(-A)^{\alpha} \Gamma_{2}^{2} B_{r}\left(0, P C_{\alpha}\left(X_{\alpha}\right)\right)$ is left equicontinuous at $t$.

Hence $\Gamma_{2}^{2} B_{r}\left(0, P C_{\alpha}\left(X_{\alpha}\right)\right)$ is equicontinuous at $t \neq t_{i}$. From these concept, we get that the operator $\Gamma^{2}$ is equicontinuous at $t \neq t_{i}$ for $i=1, \ldots, N$.

\section{Step 5.}

Next we prove that $\Gamma^{2}\left(B_{r}\left(0, P C_{\alpha}\left(X_{\alpha}\right)\right)\right)$ is continuous.

Let $\left\{u^{n}(t)\right\}_{n=0}^{\infty} \subseteq B_{r}\left(0, P C_{\alpha}\left(X_{\alpha}\right)\right)$ with $u^{(n)} \rightarrow u$ in $B_{r}$. Then there exist a number $r>0$, such that $\left\|u^{n}(t)\right\| \leq r$ for all $n$ and a.e $t \in J$, so $u^{(n)} \in B_{r}\left(0, P C_{\alpha}\left(X_{\alpha}\right)\right)$ and $u \in B_{r}$. From the defined hypothesis and if $I_{i}$ is continuous,

$$
f\left(s, u^{n}(s), \int_{0}^{s} e_{2}\left(s, \tau, u^{n}(\tau)\right) d \tau\right) \rightarrow f\left(s, u(s), \int_{0}^{s} e_{2}(s, \tau, u(\tau)) d \tau\right)
$$

for each $t \in J$, such that

$$
\left\|(-A)^{\alpha} \Gamma^{2}\left(u^{n}\left(r_{1}\right)\right)-(-A)^{\alpha} \Gamma^{2}\left(u\left(r_{2}\right)\right)\right\|
$$

$$
\leq 2 m_{f}(s) W_{f}\left\{\left\|(-A)^{\alpha} u(s)\right\|_{\alpha}+K_{e_{2}}^{*}\left\|(-A)^{\alpha} u(s)\right\|_{\alpha}\right\} .
$$

We have by the dominated convergence theorem that $\left\|(-A)^{\alpha} \Gamma^{2} u^{n}-(-A)^{\alpha} \Gamma^{2} u\right\|_{\alpha}$

$$
\begin{aligned}
= & \| \int_{0}^{t}(-A)^{\alpha} T(t-s)\left[f\left(s, u^{n}(s), \int_{0}^{s} e_{2}\left(s, \tau, u^{n}(\tau)\right) d \tau\right)\right. \\
& \left.-f\left(s, u(s), \int_{0}^{s} e_{2}(s, \tau, u(\tau)) d \tau\right)\right] d s \\
& +\sum_{0<t_{i}<t}(-A)^{\alpha} T\left(t-t_{i}\right)\left[I_{i}\left(u^{n}\left(t_{i}\right)-I_{i}\left(u\left(t_{i}\right)\right)\right] \|\right. \\
\leq & \frac{C_{\alpha}}{\left(t-t_{i}\right)^{\alpha}} \int_{0}^{t} \| f\left(s, u^{n}(s), \int_{0}^{s} e_{2}\left(s, \tau, u^{n}(\tau)\right) d \tau\right) \\
& -f\left(s, u(s), \int_{0}^{s} e_{2}(s, \tau, u(\tau)) d \tau\right) \| d s \\
& +\frac{C_{\alpha}}{\left(t-t_{i}\right)^{\alpha}} \sum_{0<t_{i}<t} \| I_{i}\left(u^{n}\left(t_{i}\right)-I_{i}\left(u\left(t_{i}\right)\right) \|\right. \\
\rightarrow & 0 \text { as } n \rightarrow \infty .
\end{aligned}
$$


EXISTENCE RESULTS FOR AN IMPULSIVE NEUTRAL

Thus $\Gamma^{2}$ is continuous. Therefore from the Arzela-Ascoli theorem, the operator $\Gamma^{2}$ is completely continuous.

In order to study the existence of mild solution of the problem $(2.1)-(2.3)$, we introduce a parameter $\lambda \in(0,1)$, and $t \in\left(t_{j}, t_{j+1}\right]$. Consider the set

$$
\begin{aligned}
G(t) & =\left\{u(t): u(t) \in \lambda(-A)^{\alpha} \Gamma u(t), 0<\lambda<1, u(t) \in P C_{\alpha}\left(X_{\alpha}\right)\right\} . \\
\frac{d}{d t}[u(t)+ & \left.\lambda g\left(t, u(t), \int_{0}^{t} e_{1}(t, s, u(s)) d s\right)\right] \\
= & A\left[u(t)+\lambda g\left(t, u(t), \int_{0}^{t} e_{1}(t, s, u(s)) d s\right)\right] \\
& +\lambda f\left(t, u(t), \int_{0}^{t} e_{2}(t, s, u(s)) d s\right), t \in(0, a], t \neq t_{i}, i=1, \ldots N \\
u(0)= & u_{0} \in X, \\
\Delta u\left(t_{i}\right)= & \lambda I_{i}\left(u\left(t_{i}\right)\right), i=1,2, \ldots N .
\end{aligned}
$$

Then by the Definition (2.1) the mild solution of the above system can be written as

$$
u(t)=\left\{\begin{array}{l}
(-A)^{\alpha} T(t)\left(u_{0}+\lambda g\left(0, u_{0}, 0\right)\right) \\
-\lambda(-A)^{\alpha} g\left(t, u(t), \int_{0}^{t} e_{1}(t, s, u(s))\right) d s \\
+\int_{0}^{t}(-A)^{\alpha} T(t-s) \lambda f\left(s, u(s), \int_{0}^{s} e_{2}(s, \tau, u(\tau)) d \tau\right) d s \\
+\sum_{t_{i}<t}(-A)^{\alpha} T\left(t-t_{i}\right) \lambda I_{i}\left(u\left(t_{i}\right)\right) \quad \forall t \in[0, a] .
\end{array}\right.
$$

The following results shows a priori bounds for the solution of the above system. $\|u(t)\|_{\alpha}$

$$
\begin{aligned}
& \leq M_{0}\left\|u_{0}\right\|+M_{0}\left\|g\left(0, u_{0}, 0\right)\right\|+K_{g}(t) \frac{\|u\|_{\alpha}}{\left(t-t_{i}\right)^{\alpha}}+\widetilde{K}_{g}(t) K_{e_{1}}^{*} \frac{\|u\|_{\alpha}}{\left(t-t_{i}\right)^{\alpha}} \\
& +K_{g}^{*}+\frac{C_{\alpha}}{\left(t-t_{j}\right)^{\alpha}} \int_{0}^{t} \frac{m_{f}(s)}{(t-s)^{\alpha}} W_{f}\left\{\left(\frac{\|u(s)\|_{\alpha}}{\left(s-t_{i}\right)^{\alpha}}\right)+K_{e_{2}}^{*}\left(\frac{\|u(s)\|_{\alpha}}{\left(s-t_{i}\right)^{\alpha}}\right)\right\} d s \\
& +\frac{C_{\alpha}}{\left(t-t_{j}\right)^{\alpha}} \sum_{i=1}^{j}\left[c_{i}\left(\frac{r}{\delta_{i-1}^{\alpha}}\right) \frac{\|u\|_{\alpha}}{\delta_{i-1}^{\alpha}}+d_{i}\right]\|u(t)\|_{\alpha} \\
& \leq \Delta+\frac{C_{\alpha}}{\left(t-t_{j}\right)^{\alpha}} \int_{0}^{t} \frac{m_{f}(s)}{(t-s)^{\alpha}} W_{f}\left\{\left(\frac{\|u(s)\|_{\alpha}}{\left(s-t_{i}\right)^{\alpha}}\right)+K_{e_{2}}^{*}\left(\frac{\|u(s)\|_{\alpha}}{\left(s-t_{i}\right)^{\alpha}}\right)\right\} d s,
\end{aligned}
$$


where $\Delta=M_{0}\left\|u_{0}\right\|+M_{0}\left\|g\left(0, u_{0}, 0\right)\right\|+K_{g}(t) \frac{\|u\|_{\alpha}}{\left(t-t_{i}\right)^{\alpha}}+\widetilde{K}_{g}(t) K_{e_{1}}^{*} \frac{\|u\|_{\alpha}}{\left(t-t_{i}\right)^{\alpha}}$ $+K_{g}^{*}+\frac{C_{\alpha}}{\left(t-t_{j}\right)^{\alpha}} \sum_{i=1}^{j}\left[c_{i}\left(\frac{r}{\delta_{i-1}^{\alpha}}\right) \frac{\|u\|_{\alpha}}{\delta_{i-1}^{\alpha}}+d_{i}\right]$.

Let us consider the right hand side of the above equaion as $v(t)$ such that $v(0)=\Delta$

$$
\begin{aligned}
v^{\prime}(t)= & \frac{C_{\alpha}}{\left(t-t_{j}\right)^{\alpha}} \frac{m_{f}(t)}{(\tau-t)^{\alpha}} W_{f}\left\{\left(\frac{\|v(t)\|_{\alpha}}{\left(t_{i}-\tau\right)^{\alpha}}\right)+K_{e_{2}}^{*}\left(\frac{\|v(t)\|_{\alpha}}{\left(t_{i}-\tau\right)^{\alpha}}\right)\right\} \\
& \quad \text { for a.e. } t \in J \\
v^{\prime}(t) \leq & B_{0} m_{f}(t) W_{f}\left(B_{1} v(t)\right)
\end{aligned}
$$

where $B_{0}=\frac{C_{\alpha}}{\left(t-t_{j}\right)^{\alpha}(\tau-t)^{\alpha}}$ and $B_{1}=\frac{1+K_{e_{2}}^{*}}{\left(t_{i}-\tau\right)^{\alpha}}$.

Then for each $t \in J$ we have

$$
\int_{v(0)}^{v(t)} \frac{d u}{B_{1} W_{f}(u)} \leq \int_{0}^{a} B_{0} m_{f}(s) d s<\int_{\Delta}^{\infty} \frac{d u}{B_{1} W_{f}(u)}
$$

Consequently there exists a constant $\bar{b}$ such that $v(t) \leq \bar{b}, t \in J$ and hence $\|u\| \leq \bar{b}$ where $\bar{b}$ depends only on the functions $W_{f}$ and $m_{f}$. This shows that $G(t)$ is bounded. We realize that $\Gamma$ has a fixed point on $J$. Therefore by the above results, the problem $(2.1)-(2.3)$ admits at least one solution on $J$.

\section{Application}

We investigate the existence of solutions for the impulsive neutral integro differential system,

$$
\begin{gathered}
\frac{d}{d t}\left[u(t, \varsigma)+\zeta_{2}(t) \mu_{1}\left(t, u(t, \varsigma), \int_{0}^{t} \mu_{2}(t, s, u(s, \varsigma)) d s\right)\right] \\
=\frac{\partial^{2}}{\partial \varsigma^{2}} u(t, \varsigma)+\zeta_{1}(t) \mu_{3}\left(t, u(t, \varsigma), \int_{0}^{t} \mu_{4}(t, s, u(s, \varsigma)) d s\right) \\
\text { where }(t, \varsigma) \in[0, a] \times[0, \pi], \\
u(t, 0)=u(t, \pi)=0, t \in[0, a] \\
u(0, \varsigma)=u(\varsigma), \varsigma \in[0, \pi] \\
u\left(t_{i}, \varsigma\right)=\theta_{i} \frac{\partial}{\partial \varsigma} u\left(t_{i}, \varsigma\right), \varsigma \in[0, \pi], i=1, \ldots, N
\end{gathered}
$$


EXISTENCE RESULTS FOR AN IMPULSIVE NEUTRAL

where $0<t_{1}<\ldots<t_{N} \leq a, \theta_{i}$ are fixed real numbers. $\zeta \in C([0, a], \mathbb{R})$, $\mu_{i}:[0, a] \rightarrow \mathbb{R}$ are suitable functions where $i=1,2,3,4$. The infinitesimal generator $\mathrm{A}$ has discrete spectrum with eigenvalues $-n^{2}, n \in N$, and associated normalised eigenvectors

$$
z_{n}(\varsigma)=(2 / \pi)^{\frac{1}{2}} \sin (n \varsigma)
$$

$\left\{z_{n}: n \in N\right\}$ denotes an orthonormal basis of $X$,

$$
T(t) x=\sum_{n=1}^{\infty} e^{-n^{2} t}\left\langle x, z_{n}\right\rangle z_{n}
$$

such that $\|T(t)\| \leq e^{-t}$ for $x \in X, t \geq 0$,

$$
\begin{aligned}
(-A)^{\frac{-1}{2}} x & =\sum_{n=1}^{\infty} \frac{1}{n}\left\langle x, z_{n}\right\rangle z_{n} \text { for } x \in X, \\
(-A)^{\frac{1}{2}} x & =\sum_{n=1}^{\infty} n\left\langle x, z_{n}\right\rangle z_{n} \text { for } x \in D\left((-A)^{\frac{1}{2}}\right) \\
& =\left\{x \in X: \sum_{n=1}^{\infty} n\left\langle x, z_{n}\right\rangle z_{n} \in X\right\}, \\
\text { and }\left\|(-A)^{-1 / 2} T(t)\right\| & \leq \frac{1}{\sqrt{2}} e^{t / 2} t^{-1 / 2} \quad \forall t>0
\end{aligned}
$$

We assume $\zeta_{1}, \zeta_{2}, \widetilde{\zeta}_{1}, \widetilde{\zeta}_{2} \in L^{q^{\prime}}([0, a] ; \mathbb{R})$ for few $q \in[0,2)$ such $q^{\prime}$ represents the conjugate of $q$. We initiate the functions $f, g:[0, a] \times X \times X \rightarrow X$ and $I_{i}=X_{1 / 2} \rightarrow X$ by

$$
\begin{aligned}
& f(\cdot, \varphi, \mu)=\zeta_{1}(t) \mu_{3}\left(t, u(t, \varsigma), \int_{0}^{t} \mu_{4}(t, s, u(s, \varsigma)) d s\right) \\
& g(\cdot, \vartheta, \sigma)=\zeta_{2}(t) \mu_{1}\left(t, u(t, \varsigma), \int_{0}^{t} \mu_{2}(t, s, u(s, \varsigma)) d s\right)
\end{aligned}
$$

and $I_{i}: X_{1 / 2} \rightarrow X$ is given by $I_{i}(x)=\theta_{i} \frac{\partial}{\partial \varsigma} u\left(t_{i}, \varsigma\right)$, This will imply that $I_{i} \in L\left(X_{1 / 2}, X\right)$ for every $i$. Then there exists a constant $C>0$ such that $\left\|I_{i}\right\|_{L\left(X_{1 / 2}, X\right)} \leq C\left|\theta_{i}\right|$ for all $i=1, \ldots, N$. Define

$$
\omega:=\gamma+\frac{2^{\frac{1}{2}+\frac{1}{q}}}{\left|1-\frac{q}{2}\right|^{\frac{1}{q}}}
$$


EXISTENCE RESULTS FOR AN IMPULSIVE NEUTRAL

$$
\frac{1}{\sqrt{2}}\left\{p_{1}+\Upsilon+C \sum_{i=1}^{N} \frac{\left|\theta_{i}\right|}{\delta_{i-1}^{\frac{1}{2}}}\right\}<1
$$

where

$$
\Upsilon=\left\{\left(\left\|\zeta_{1}\right\|_{L^{q^{\prime}}\left(\left[0, t_{j}\right]\right)}+\mu_{4}^{*}\left\|\widetilde{\zeta}_{1}\right\|_{L^{q^{\prime}}\left(\left[0, t_{j}\right]\right)}\right) \omega\left[\frac{2^{\frac{1}{2} a^{\frac{1}{q}-\frac{1}{2}}}}{\left(1-\frac{q}{2}\right)^{\frac{1}{q}}}\right]\right\},
$$

and for $c>0$

$$
p_{1}=\left[c \zeta_{2}(t) \frac{\left(t-t_{j}\right)^{\frac{1}{2}}}{C_{\frac{1}{2}}\left(t-t_{i}\right)^{\frac{1}{2}}}+c \widetilde{\zeta}_{2}(t) \mu_{2}^{*} \frac{\left(t-t_{j}\right)^{\frac{1}{2}}}{C_{\frac{1}{2}}\left(t-t_{i}\right)^{\frac{1}{2}}}\right]
$$

where

$$
\mu_{k}^{*}=\max _{t \in J} \int_{0}^{t} \mu_{k}(t, s) d s, k=2,4
$$

Proposition 4.1. Assume that $\frac{1}{\sqrt{2}}\left\{p_{1}+\Upsilon+C \sum_{i=1}^{N} \frac{\left|\theta_{i}\right|}{\delta_{i-1}^{\frac{1}{2}}}\right\}<1$. Then there exists a unique mild solution $u \in P C_{\frac{1}{2}}\left(X_{\frac{1}{2}}\right)$ of (4.1)-(4.4).

Proof. By Corollary 3.1, it follows that $u \in P C_{\frac{1}{2}}\left(X_{\frac{1}{2}}\right)$ is a mild solution of (4.1) - (4.4).

\section{References}

[1] A. Anguraj, M. Mallika Arjunan and E. Hernandez, Existence results for an impulsive neutral functional differential equation with state-dependent delay, Applicable Analysis, 86(7)(2007), 861-872.

[2] A. Anguraj and K. Karthikeyan, Existence of solutions for impulsive neutral functional differential equations with nonlocal conditions, Nonlinear Analysis: Theory, Methods $\&$ Applications, 70(7)(2009), 2717-2721.

[3] K. Balachandran and N. Annapoorani, Existence results for impulsive neutral evolution integrodifferential equations with infinite delay, Nonlinear Analysis: Hybrid Systems, 3(2009), 674-684.

[4] M. Benchohra, J. Henderson and S. Ntouyas, Impulsive Differential Equations and Inclusions, Hindawi Publishing Corporations, Contemporary Mathematics and Its Applications, Volume 2, 2006. 
EXISTENCE RESULTS FOR AN IMPULSIVE NEUTRAL

[5] T. Cardinali and P. Rubbioni, Impulsive semilinear differential inclusion: Topological structure of the solution set and solutions on non-compact domains, Nonlinear Analysis: Theory, Methods \& Applications, 69(1)(2008), 73-84.

[6] A. Chadha and Dwijendra N Pandey, Existence results for an impulsive neutral integro-differential equation with infinite delay via fractional operators, Malaya Journal of Matematik 2(3)(2014), 203-214.

[7] Y.K. Chang, A. Anguraj and M. Mallika Arjunan, Existence results for impulsive neutral functional differential equations with infinite delay, Nonlinear Analysis: Hybrid Systems, 2(1)(2008), 209-218.

[8] Y.K. Chang, A. Anguraj and M. Mallika Arjunan, Existence results for non-densely defined neutral impulsive differential inclusions with nonlocal conditions, Journal of Applied Mathematics and Computation, 28(1)(2008), 79-91.

[9] Y.K. Chang, A. Anguraj and K. Karthikeyan, Existence for impulsive neutral integro-differential inclusions with nonlocal initial conditions via fractional operators, Nonlinear Analysis: Theory, Methods \& Applications, 71(10)(2009), 4377-4386

[10] K. Ezzinbi, Xianlong Fu and Khalid Hilal, Existence and regularity in the $\alpha$ - norm for some neutral partial differential equations with nonlocal conditions, Nonlinear Analysis: Theory, Methods $\&$ Applications, 67(5)(2007), 1613-1622.

[11] Z. Fan and G. Li, Existence results for semilinear differential equations with nonlocal and impulsive conditions, Journal of Functional Analysis, 258(5)(2010), 1709-1727.

[12] J.K. Hale, Partial neutral functional-differential equations, Romanian Journal of Pure and Applied Mathematics, 39(4)(1994), 339-344.

[13] J.K. Hale and S.M. Verduyn Lunel, Introduction to FunctionalDifferential Equations, Applied Mathematical Sciences, Springer-Verlag, New York, 99(1993).

[14] E. Hernandez and Donald O'Regan, Existence results for a class of abstract impulsive differential equations, Bulletin of Australian Mathematical Society, 87(3)(2013), 366-385. 
EXISTENCE RESULTS FOR AN IMPULSIVE NEUTRAL

[15] E. Hernandez, Jair Silverio dos Santos and Katia A.G. Azevedo, Existence of solutions for a class of abstract differential equations with nonlocal conditions, Nonlinear Analysis: Theory, Methods \&6 Applications, 74(7)(2011), 2624-2634.

[16] E. Hernandez and Donald O'Regan, On a new class of abstract neutral differential equations, Journal of Functional Analysis, 261(12)(2011), $3457-3481$.

[17] E. Hernandez and H.R. Henriquez, Impulsive partial neutral differential equations, Applied Mathematics Letters, 19(3)(2006), 215-222.

[18] L. Hu and Y. Ren, Existence results for impulsive neutral stochastic functional Integro-differential equations with infinite delay, Acta Applicandae Mathematicae, 111(3)(2010), 303-317.

[19] V. Lakshmikantham, D. Bainov and P.S. Simeonov, Theory of Impulsive Differential Equations, World Scientific, Singapore, (1989).

[20] N.Y. Nadaf and M. Mallika Arjunan, Existence and controllability results for damped second order impulsive neutral functional differential systems with state-dependent delay in Banach spaces, Malaya Journal of Matematik, 1(1)(2013), 70-85.

[21] A. Pazy, Semigroups of Linear Operators and Applications to Partial Differential Equations, Springer-Verlag, New York, 44, 1983.

[22] C. Parthasarathy and M. Mallika Arjunan, Existence results for impulsive neutral stochastic functional integro-differential systems with infinite delay, Malaya Journal of Matematik 1(1)(2012), 26-41.

[23] S. Selvarasu, P. Kalamani and M. Mallika Arjunan, Approximate controllability of nonlocal impulsive fractional neutral stochastic integrodifferential equations with state-dependent delay in Hilbert spaces, Malaya Journal of Matematik, 4(4)(2016), 571-598.

[24] A. Vinodkumar, Existence and uniqueness of solutions of random impulsive differential equations, Malaya Journal of Matematik, 1(1)(2012), 8-13.

[25] J. Wu and H. Xia, Rotating waves in neutral partial functional-differential equations, Journal of Dynamics and Differential Equations, 11(2)(1999), 209-238. 
EXISTENCE RESULTS FOR AN IMPULSIVE NEUTRAL

[26] J. Wu and H. Xia, Self-sustained oscillations in a ring array of coupled loss less transmission lines, Journal of Differential Equations, 124(1)(1996), $247-278$.

[27] J. Wu, Theory and Applications of Partial Functional-Differential Equations, Applied Mathematical Sciences, Springer- Verlag, Newyork, 119, 1996.

Venkatesh Usha,

Department of Mathematics,

PSG College of Arts \& Science,

Coimbatore - 641 014, Tamil Nadu, India

Email: usha@psgcas.ac.in

Dumitru Baleanu,

Department of Mathematics,

Faculty of Art and Sciences,

Cankaya University, 06530 Ankara,

Turkey and Institute of Space Sciences,

Magurele-Bucharest, Romania.

Email: dumitru@cankaya.edu.tr

Mani Mallika Arjunan,

Department of Mathematics,

C. B. M. College, Kovaipudur,

Coimbatore - 641 042, Tamil Nadu, India.

Email: arjunphd07@yahoo.co.in 